\title{
Experiences using CAS and multimedia in teaching vectorcalculus
}

\author{
ILDIKÓ PERJÉSI-HÁMORI
}

Abstract. The development of informatics brings new opportunities that need reevaluating of the teaching concepts. For this reason we have performed a comprehensive educational development for engineering students. Our main goals were to work out a new educational strategy, to develop the needed package of the subject material, to introduce the strategy in the practice, to analyze and evaluate the experiences. In the developed and adapted teaching-learning strategy the teacher is the organizer, designer and the manager of the process. In this paper we summarize the concepts, the results and experiences of the 3-years-long development.

Key words and phrases: computer algebra and multimedia in teaching-learning process, didactical questions of CAS.

ZDM Subject Classification: B45, C75, U55.

\section{Introduction}

Learning at university or college in the last centuries meant that professors interpreted the science, as they knew it, and students tried to understand, learn, and apply it. The types of lessons were separate lectures, seminars and laboratory practices. With this teaching style, relatively few people got chance to learn in higher education.

The demands of nowadays higher education significantly increased these possibilities:

- More and more people get the opportunity to learn at university level. For

Copyright (C) 2003 by University of Debrecen 
example, Hungary would like to reach that half of people graduated in high schools should continue their studies at universities.

- People are pressed and encouraged to life-long learning while they have to get acquainted with new professions besides their everyday works.

- The Bologna agreement prefers the two-level higher educational system in Europe. It requires unified and well-defined curricula at both levels.

- The students come into the engineering faculties without entrance examination, with very different basic knowledge levels. This fact brings a lot of problems, first of all in the teaching of the basic subjects, such as mathematics and mechanics.

- Because of the educational policy, which requires "effective" educational methods, the numbers of contact lessons have been decreased; meanwhile the student/educator rate was constantly increased.

- The recent great developments of informatics brings new opportunities that need a complete reevaluating of the teaching concepts. A lot of methods have been developed to acquire the knowledge which is dispersed in the world [1]-[8].

In this changing world the most important task for the teaching stuff is to improve and control the teaching-learning process.

For the above mentioned reasons we have performed a comprehensive educational development for mechanical engineering students for the course vectorcalculus (Mathematics III). In the following we summarize the results and experiences of this 3-years-long improvement.

\section{Goals and methods of development}

The main goals of our research teaching Mathematics III to mechanical engineer students were to:

- work out a new educational strategy,

- develop the needed package of the subject material,

- introduce the strategy into the practice,

- analyze the experience from scientific point of view of the mathematical didactics,

- evaluate of the attained results. 
Our mathematical goals were to teach students to be able to apply the scalarvector, the vector-scalar and vector-vector functions, operations of vector functions, and the Gauss- and Stokes theorems in the engineering practice.

While developing the method we took into account the opportunities of the modern techniques (Internet and computer algebra systems) and results of the new methodological research (particularly the open learning and the mathematical didactics).

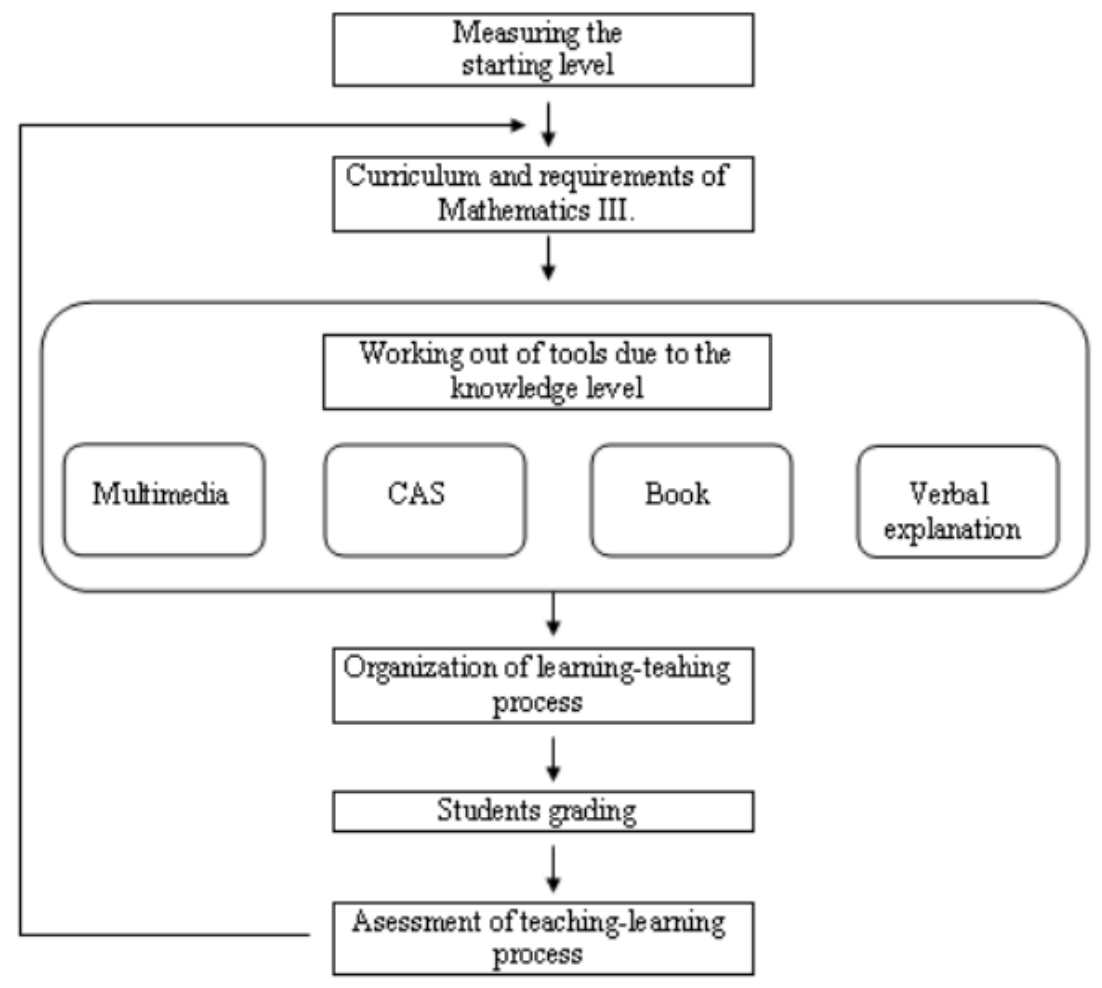

Figure 1. Steps of the development

The steps of the development are shown on the Figure 1. It was a one-group experiment, where the starting position was represented by the students' knowledge checked by a repetitive test and the Mathematics II grades of the members of the group. (The requirement to take Mathematics III was the completion of Mathematics II.) To control the teaching-learning process we checked the acquired knowledge by a test containing theoretical questions and practical problems. The 
teacher was the organizer, designer and the manager of the teaching-learning process.

The teacher

- chooses the strategy,

- controls the process,

- is the expert of the subject matter,

- knows and selects - in accordance with the intellectual operations and inner mental processes - the presentation manners and tools of the subject material,

- is familiar with the students preliminary knowledge,

- emphasizes the main topics of the curriculum - with help of verbal explanation and guided problem solving - on the contact lessons,

- keeps contact with students through e-mail,

- evaluates the developments of students knowledge, modify the strategy.

To introduce and assess the new teaching-learning strategy we carried out an educational research. The independent variables of the experiment were the subject material system, which was developed by us to teach vectorcalculus, and the educational strategy carried out by means of the subject material. The dependent variables of the experiment were the students' subject knowledge level and the adequate achievement.

\section{Preparatory steps}

\subsection{CAS in Mathematics II}

As preparatory steps of the new educational form, we applied the visualization features of MAPLE computer algebra system (CAS) in the lectures of Mathematics II, and we made the prepared worksheets about differential- and integral calculus of one- and two variable functions available on the local network. This way, the students who were interested in these new possibilities could get acquainted them in advance.

\subsection{Measuring the students' knowledge level before the experiments}

To sum up the initial state we prepared a written repetitive test to check the knowledge on topics of Mathematics II. Later, we linked this test to the Internet based subject material to make self-control possible. While evaluating 
the results we found that the knowledge of 25 percents of the full-time students and 50 percents of distance learning students didn't reach the sufficient level (the 40 percent of the maximum point).

\subsection{Language knowledge}

Because there is only the English version of the MAPLE software available, the second element of the initial state was testing the English knowledge of the students. Unfortunately, most of them didn't have the proper level of English to understand the commands of the software, this fact was critical that we had to take into account in the development of materials.

\subsection{Learning style}

Another part of the initial state aimed at mapping the learning style of the students. We based this examination on D. A. Kolb's self-controlling test [9]. The result confirmed the hypothesis that the learning habit of engineering students shifted towards the concrete experience-based learning, while the active and passive learning styles are practically in balance. From these facts we drew the conclusion, that the inductive teaching-learning method should be preferred to the deductive ones for engineering students (Figure 2). This fact is important to know for the teachers, because their learning style is more abstract as their students.

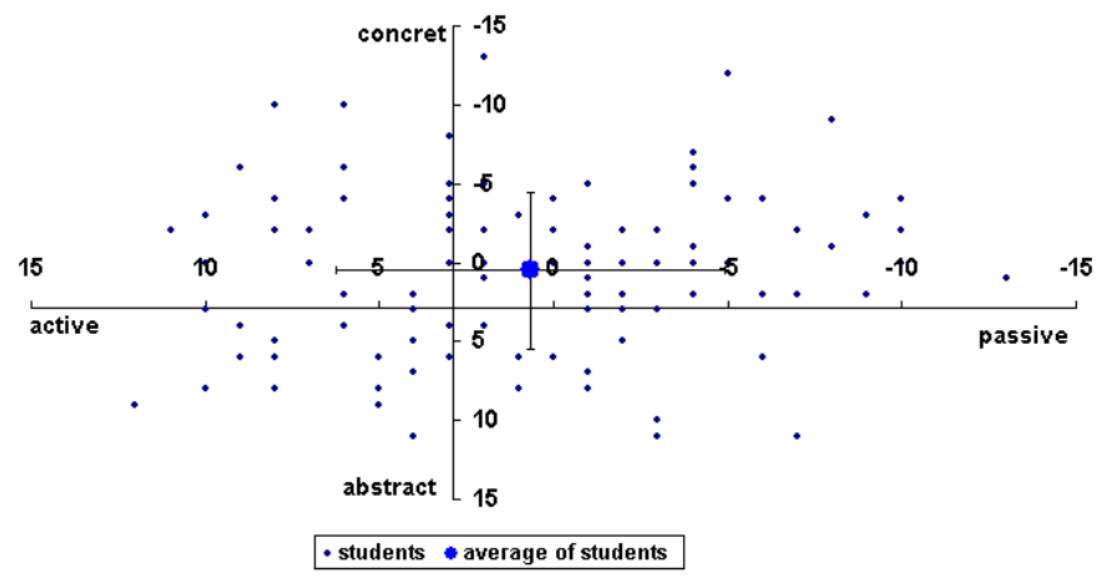

Figure 2. Learning styles of mechanical engineering students 


\section{Interactive learning environment}

Our tools used in the teaching-learning process of vectorcalculus were multimedia, computer algebra system, textbook and verbal explanation. In this session we show some examples from the vectorcalculus to illustrate the advantage of the new computer-based teaching-learning strategy.

\subsection{Multimedia}

We used multimedia tools to illustrate the physical meaning of mathematical concepts and to process the subject material of the course. The web-based syllabus is available on the web [10], [11]. It contains 10 lessons, multiple choice tests, self-controlling questions, MAPLE worksheets in html format, downloadable MAPLE worksheets and information about the course. The advantage of the webbased materials is the use of colors, navigation, hyperlink, multiple choice tests, new forms of visualization, interactivity and the possibility of dynamic development.

\subsection{CAS}

We used the visualization features of CAS mostly to introduce new types of mathematical objects. With the help of analytical possibility of CAS such concepts could be demonstrated that could not have been done by using only the classical tools. By means of this tool, accommodation of the new concepts and completion of the old, classical schemes become easier.

Example 1. Plot the elements of the vector field that are localized on the curve.

$$
\text { field }:=[-y, x, z], \quad \text { curve }:=\left[x=\cos (t)^{2}, y=\cos (t) \sin (t), z=\sin (t)\right]
$$

We demonstrate the result of a written procedure on Figure 3, which makes the opportunity for the user to illustrate the localized elements of the vector field into the curve. 

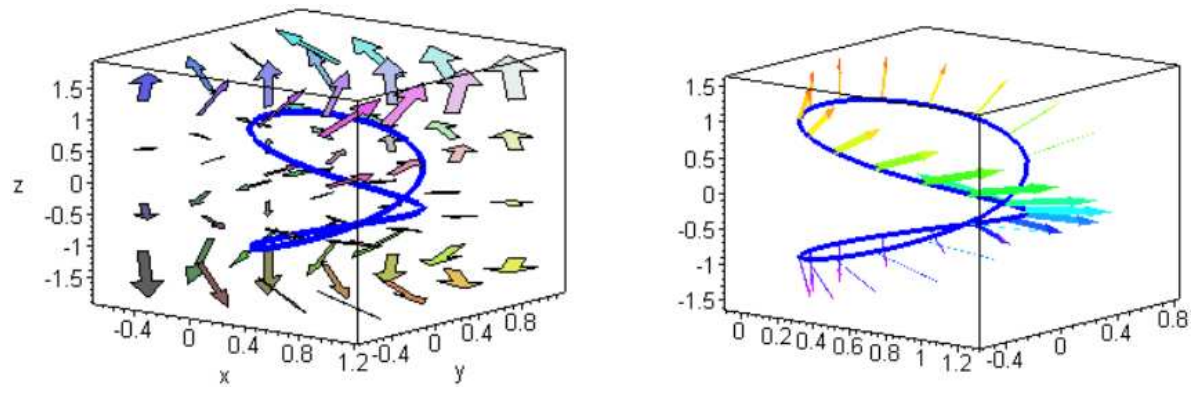

Figure 3. Visualization of the Viviani-curve and a vector field together

To teach vectorcalculus the numerical calculation possibilities of CAS was very useful to substitute the time-consuming and difficult numerical hand-operated calculations. With the help of it we could develop the students' ability of analogy, comparison, generalization and assimilation.

We used symbolical calculations with CAS mainly to prove identities. It has outstanding role to form the ability of abstraction.

Example 2. Prove the identity $\nabla \cdot(\boldsymbol{a} \times \boldsymbol{b})=\boldsymbol{b} \cdot(\nabla \times \boldsymbol{a})-\boldsymbol{a} \cdot(\nabla \times \boldsymbol{b})$ if a and $\mathrm{b}$ are vector fields.

$>\operatorname{alias}(\mathrm{a}=[\mathrm{a} 1(\mathrm{x}, \mathrm{y}, \mathrm{z}), \mathrm{a} 2(\mathrm{x}, \mathrm{y}, \mathrm{z}), \mathrm{a} 3(\mathrm{x}, \mathrm{y}, \mathrm{z})]) ; \mathrm{alias}(\mathrm{b}=[\mathrm{b} 1(\mathrm{x}, \mathrm{y}, \mathrm{z}), \mathrm{b} 2(\mathrm{x}, \mathrm{y}, \mathrm{z}), \mathrm{b} 3(\mathrm{x}, \mathrm{y}, \mathrm{z})])$;

$>$ left := diverge $(\operatorname{crossprod}(a, b),[x, y, z])$;

$>\operatorname{right}:=\operatorname{dotprod}\left(b, \operatorname{curl}(a,[x, y, z]),{ }^{\prime} \operatorname{orthogonal}{ }^{\prime}\right) \operatorname{dotprod}\left(a, \operatorname{curl}(b,[x, y, z]),{ }^{\prime}\right.$ orthogonal $\left.{ }^{\prime}\right)$;

>simplify(right-left);

The result is 0 (zero), which means that the statement was true. To prove it without CAS is a very hard and time-consuming job without using new ideas. During the application of CAS we followed the didactical principles.

a) The developed CAS applications had to cover the whole syllabus of the course. The worksheets of the course are organized into one system by means of the "Table of Contents" MAPLE worksheet. In this page there are hyperlinks to the exercises and the exercises end up with a hyperlink to the main sheet. So there is a close connection between the parts of the course.

b) The user must have the opportunity to get familiar with the special language of CAS (MAPLE). We allowed only the most necessary commands to be used. 
Example 3. Find the tangent plane of the surface in the given point.

The algorithm of the solution:

Definition of the surface and the given point:

$>\mathrm{r}(\mathrm{u}, \mathrm{v}):=\left[\cos (\mathrm{u})-\mathrm{v}^{*} \sin (\mathrm{u}), \sin (\mathrm{u})-\mathrm{v}^{*} \cos (\mathrm{u}), \mathrm{u}+\mathrm{v}\right]$;

$>r 0:=\operatorname{simplify}(\operatorname{subs}(u=0, v=1, r(u, v)))$;

Determining the partial derivatives:

$>\mathrm{ru}:=\operatorname{diff}(\mathrm{r}(\mathrm{u}, \mathrm{v}), \mathrm{u}) ;>\mathrm{rv}:=\operatorname{diff}(\mathrm{r}(\mathrm{u}, \mathrm{v}), \mathrm{v})$;

Finding the value of the partial derivatives in the given point:

$>\mathrm{u} 0:=0 ; \mathrm{v} 0:=1 ;>\mathrm{ru} 0:=\operatorname{simplify}(\operatorname{subs}(\mathrm{u}=\mathrm{u} 0, \mathrm{v}=\mathrm{v} 0, \mathrm{ru}))$;

$>$ rv0 $:=\operatorname{simplify}(\operatorname{subs}(u=u 0, v=v 0, r v))$;

Calculating of the cross product of the received values, which will be the normal vector of the tangent plane. (First new command: crossprod)):

$>\mathrm{n}:=\operatorname{crossprod}(\mathrm{ru} 0, \mathrm{rv} 0)$;

Writing out the equation of the tangent plane. (Command: dotprod):

$>\mathrm{R}:=[\mathrm{x}, \mathrm{y}, \mathrm{z}] ;>$ planeequation $:=\operatorname{dotprod}(\mathrm{R}-\mathrm{r} 0, \mathrm{n})=0$;

In this exercise-solving method there were only two new commands, which didn't disturb the mathematical problem-solving. The worksheet contained textual explanations but it avoided the extreme comments. The worksheet introduced every new command - according to the principle of spirality - through a mathematical problem.

c) The CAS worksheets were written considering the gradation principle.

Example 4. Calculate the line integral of the vector field on a given curve.

Definition of the field:

>'field' :=[x^2*y,x-z, $\left.x^{*} y^{*} z\right]$;

Definition of the curve:

$>$ 'curve': $=\left[x=t, y=t^{\wedge} 2, z=2\right]$;

Localization of the field elements into the curve:

>'localization':= subs('curve','field');

Determination of the tangent of the curve:

>'tangent' := [diff(rhs('curve'[1]),t), diff(rhs('curve'[2]),t), diff(rhs('curve'[3]),t)];

Scalar product of 'localization' end 'tangent':

>'dotprod ' = dotprod('localization','tangent', orthogonal); 
Integration the result in the given parameter range:

$$
\begin{gathered}
>\text { 'Lineintegral }{ }^{\prime}=\operatorname{lnt}(\operatorname{rhs}(\%), \mathrm{t}=0 . .2) ; \operatorname{value}(\%) \\
\text { Lineintegral }=\int_{0}^{2} t^{4}+2 t(t-2) d t \text { Lineintegral }=\frac{56}{15}
\end{gathered}
$$

After execution of the above commands we can write a procedure, which contains them. Afterwards the procedure can be used to execute a line integral.

$>$ lineintegral := proc(field,curve,t1,t2)

local tangent,skalarszorzat, integrandus, integral,lok;

tangent $:=\operatorname{diff}(\operatorname{map}($ rhs,curve $), \mathrm{t})$;

lok:= subs(curve,field);

skalarszorzat:= dotprod(tangent,lok,orthogonal);

integrandus : = simplify(skalarszorzat);

integral := Int(integrandus, $\mathrm{t}=\mathrm{t} 1 . . \mathrm{t} 2)$; integral $=$ value $(\%)$;

end:

We can call this procedure for optional vector field, curve and parameter range.

$$
\begin{aligned}
>\text { Lineintegral }:=\text { lineintegral('field','curve',0,2); } \\
\text { Lineintegral }:=\int_{0}^{2} t^{4}+2 t^{2}-4 t d t=\frac{56}{15}
\end{aligned}
$$

We detailed all steps of problem-solving on those exercises that we could solve without the computer algebra system. We wrote the procedures by means of frequently used commands only after their detailed explanations. (White box black box effect.)

d) We used the black box - white box effect on those commands that are used only for visualization, or rather where the algorithm of the solution passes the frame of the course. It means there is an opportunity to understand each step of the solution for those students who want to do so. But everybody knows what the scheme of the problem solving is and what the result is of the procedures (principle of the operationalization).

Example 5. Plot the trajectory curves of a vector field.

To execute this problem, we have to solve a differential equation system. The curriculum of the mathematics courses do not contains this kind of problemsolving 
The vector field and the particular solutions (trajectories) of the differential equation system, according with different starting assumptions, is shown on Figure 4. The process of problem-solving is clear without getting to know all steps of the solution.

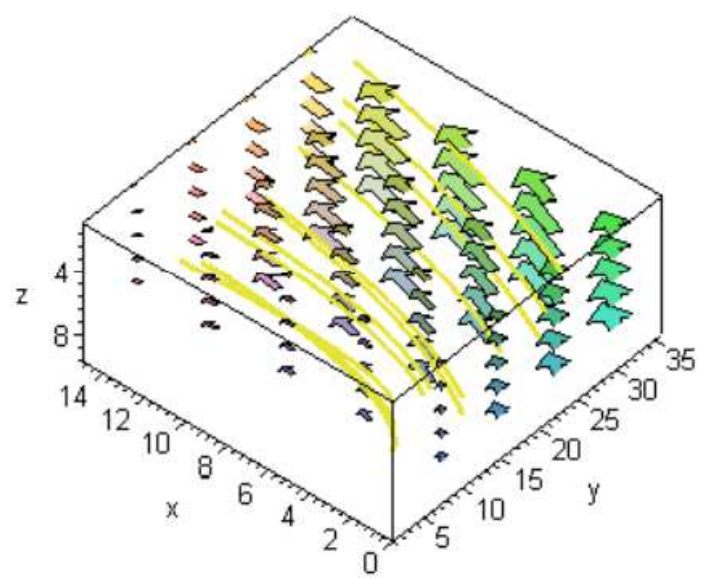

Figure 4. Vector field and its trajectory curves

It is important to profit the graphical opportunity in each exercise; to help the concept-meaning not only at the symbolical but at the image level as well

e) We had to avoid using CAS only for itself; it is only a tool of the mathematical subject.

f) The worksheets contained exercises to be solved by the user alone.

g) We had to take into account that some hard exercises became routine ones with help of it.

Example 6. Determination of the surface integral.

Definition of the vector field:

$>$ field : $=[x-2 * y, z+x, 3]$;

Definition of the surface. It is important to emphasize that the values of $x$, $y, z$ variables are on the given surface.

$>$ surface $:=[x=(1+2 * \cos (u)) * \cos (v), y=(1+2 * \cos (u)) * \sin (v), z=2 * \sin (u)]$;

Partial derivatives

$>$ ru := diff(map(rhs,surface),u); >rv := diff(map(rhs,surface),v); 
Their cross production $>\mathrm{n}:=\operatorname{crossprod}(\mathrm{rv}, \mathrm{ru})$;

Localization of the field elements into the surface: $>$ lok:=subs(surface, field);

Scalar product of $n$ and lok:

>integrandusz : = simplify(dotprod(lok, $\mathrm{n}$, orthogonal));

Double integral of the above result:

$>$ Surfaceintegral $=$ Doubleint $($ integrandusz, $\mathrm{u}=0 . . \mathrm{Pi}, \mathrm{v}=0 . .2 * \mathrm{Pi}) ;$ value $(\%)$;

h) While planning the worksheets we paid attention to the principle of consciousness due to the fact that it is not the technical details but the mathematical meaning is always the most important. Our goal was that the student-users could adopt the concepts and procedures into new situations.

Example 7. Conjecture of the Stokes theorem

The algorithm of the solution:

Definition of the field and the curve:

$$
\text { field }:=[y, z, x] \text { circle }:=[2 \sin (t), 2 \cos (t), 3]
$$

Demonstration of the field and the curve:

Writing a procedure, which plots optional field and curve.

Writing a procedure, which plots only the localized field elements and curve (Figure 5).
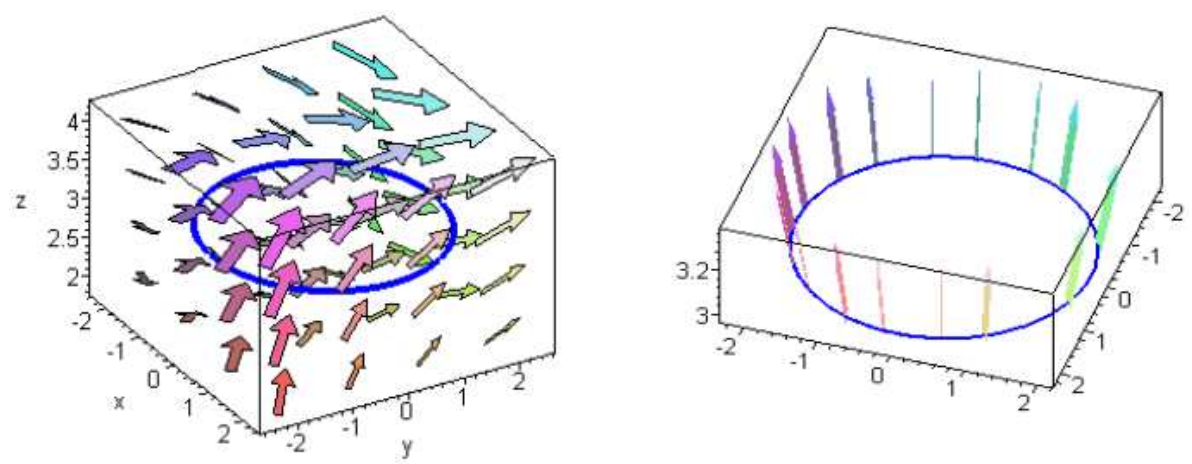

Figure 5. Vector field and curve. 
Determination of the line integral:

$$
\text { Lineintegral }:=\int_{0}^{2 \pi} 4 \cos \left(t^{2}\right)-6 \sin (t) d t=4 \pi
$$

Determination of the curl of the vector field:

$$
\operatorname{curl}:=[-1,-1,-1]
$$

Specification of the first surface (hemisphere), which is enclosed by the given curve (Figure 6a):

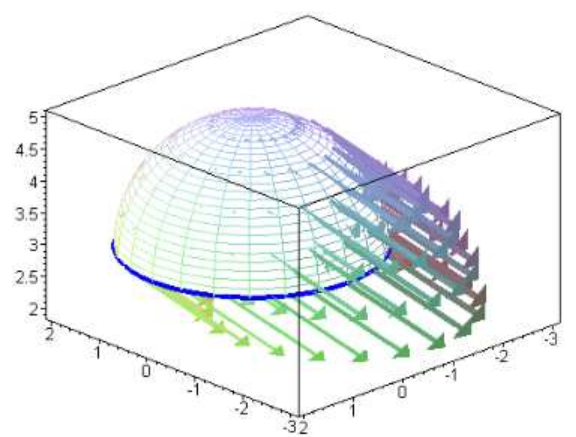

Figure 6a. Hemisphere, circle, curl of field

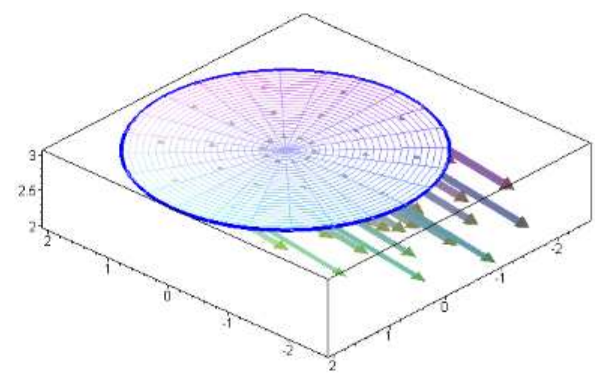

Figure 6b. Disk, circle, curl of field

Determination of the surface integral of the curl on the hemisphere:

$$
\begin{aligned}
& \text { Surface_integral1 := } \\
& \qquad \int_{0}^{1 / 2 \pi} \int_{0}^{2 \pi} 4 \sin (v)(\cos (u) \sin (v)+\sin (u) \sin (v)+\cos (v)) d u d v=4 \pi
\end{aligned}
$$

Determination of the surface integral on the disk (Figure 6b):

$$
\text { Surface_integral2 }:=\int_{0}^{2 \pi} \int_{0}^{2} u d u d v=4 \pi
$$

Conjecture of the Stokes theorem.

After the self-supporting exercises the generalization could come. It states that under the same conditions the vector-vector function's line integral on a closed curve and the surface integral of curl of this vector-vector function are equal when the curve is the boundary of the surface. 


\subsection{Book}

The textbook [12] in this system is a supplemental tool. It is used for those students, who wanted to know more about the course subjects. For example, it contains more physical applications, which are not the basic task of the curse. On the other hand, we detailed here the exact proofs of those theses which were proved on the contact lessons only heuristically. Writing the book we took into account the recommendations of open learning methodology.

\subsection{Verbal explanation}

Verbal explanation is definitely necessary at introduction of new concepts and demonstration of new type of exercises through easy examples. It doesn't mean only a simple fact delivery; however, it means consultation about the revealed problems. The exploration of connections, abstraction, and generalization requires direct professorial control in higher education as well. The amount of verbal explanation depends of the students' demands but the role of it in the accommodation is never negligible.

\section{Didactical analysis and evaluation of development}

\subsection{Evaluation of the interactive learning environment}

According to our experience, the interactive learning environment:

- has the great advantage of flexibility and interactivity. It makes possible that the prepared worksheets and web pages are changing and becoming more correct from lesson to lesson based on the experience of the user (student and teacher),

- is especially important in development of teaching of engineers because in engineering the computer usage has great significance,

- doesn't substitute the exact definitions of mathematical concepts and procedures but it is used to assist to formulate and apply them.

For the mathematical problem-solving, CAS is an important tool in the teachinglearning process. CAS:

- has got the advantage that the user can concentrate on the main concepts, his attention is not distracted by extra calculations,

- has important role in exact problem definition, 
- helps the user to get practice in elementarization of investigated problems and organize the elements into a system. This way, CAS helps to build new thinking schemes and to impregnate them into old structures,

- usage is effective only for small students' groups in a computer laboratory,

- needs more contact lessons to learn the basic concepts but it is paid off when the exercise solving becomes easier,

- effectiveness increases if its application is proceeded by simple exercise-solving by chalk-and-talk under teacher's control.

\subsection{Comparative analysis of students' achievements}

To facilitate the continuous learning - instead of the examination at the end of the semester - we checked the acquired knowledge by two semester tests on theoretical questions (without computer) and practical topics (with computer). The passing level was 50 percent in the theoretical questions and 40 percent in the practices.

We can see on the Figure 7 that there are approximately four times as many students who successfully achieved Mathematics III as many don't. On the contrary, these rates were $2 / 5$ and $3 / 5$, respectively on Mathematics II course.

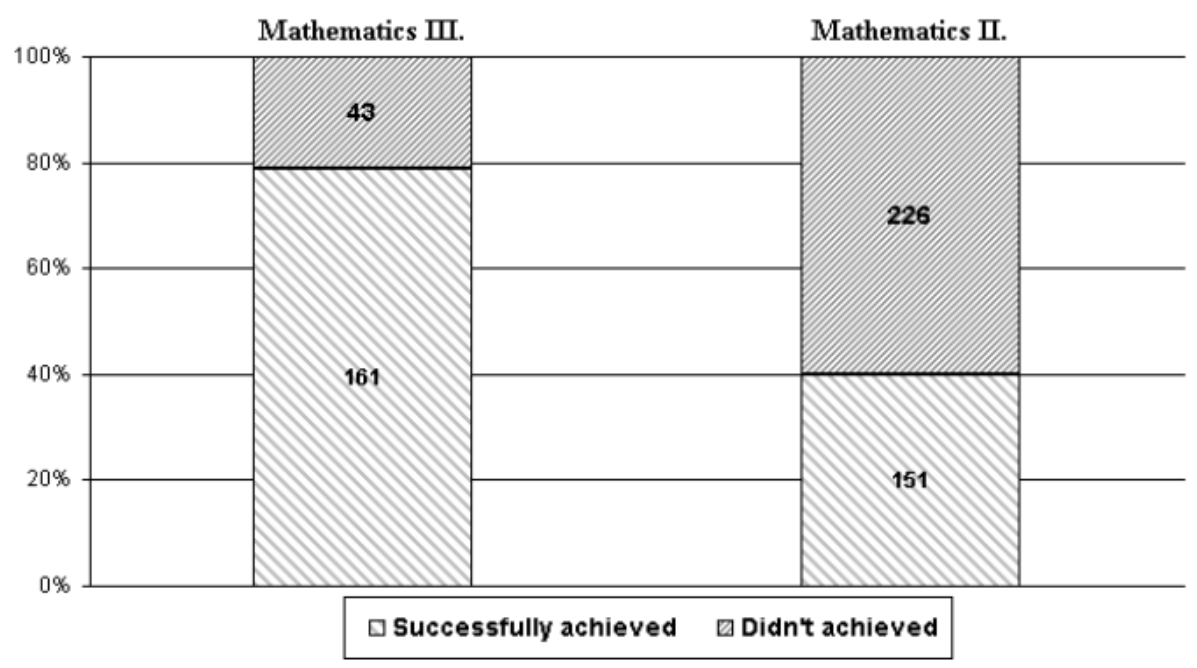

Figure 7. Successfully achieved student's rate to subject picked up students 


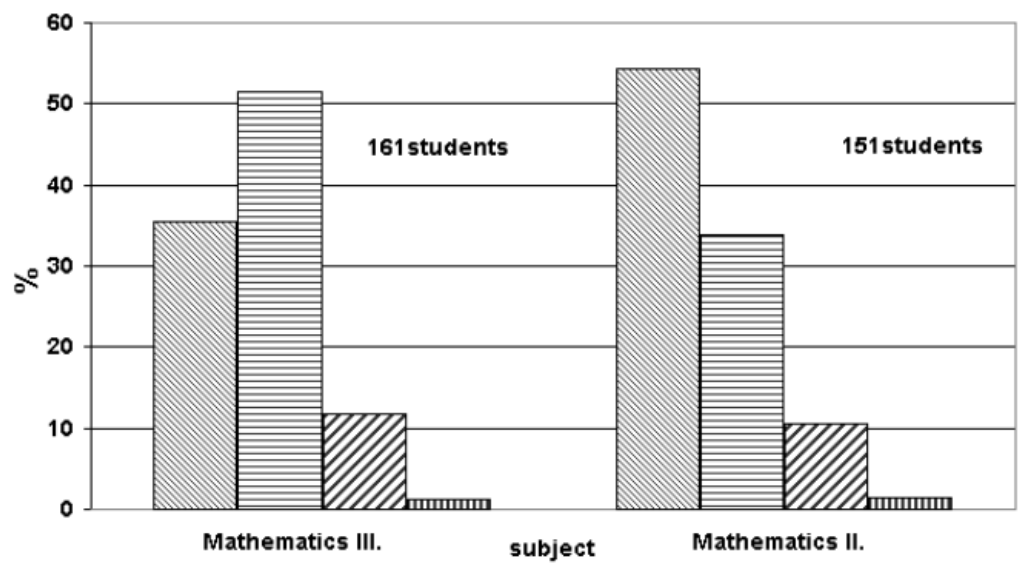

Figure 8. Marks of the students who successfully achieved Mathematics III.

Figure 8 shows that distribution of the successful students' marks shifted toward the mark 3 (average).

We performed statistical evaluation to decide whether the difference is significant or not. We determined the correlation coefficients of the marks $(r)$, and with help of Student's t-test and $\chi^{2}$ test the significance level of the data. Table 1 shows the calculated data and the data from the reference table; $p$ means the level of probability.

\begin{tabular}{l|c|c|c}
\hline Value & $\boldsymbol{r}$ & $\boldsymbol{t}$ & $\chi^{2}$ \\
\hline calculated & 0.213 & 5.590 & 15.925 \\
\hline from literature & $0.208(p<0.01)$ & $2.609(p<0.01)$ & $14.685(p<0.1)$ \\
\hline
\end{tabular}

Table 1. Statistical connection between marks of Mathematics II and Mathematics III

It is shown in the Table 1 that the correlation coefficient is a little bit higher than the literature value to 150 independence degrees, so there is a connection between the two sets of data. It is not a surprise, since the data refer to the same students' group. The value of the one-pattern $t$ test shows that the marks of Mathematics III were significantly higher, supposing $1 \%$ of error level. The $\chi^{2}$ test value means that the values are significantly different at $10 \%$ error level.

We compared the results of the theoretical tests and practical tests, and performed the statistical calculations with these data as well from Mathematics III. 
Unfortunately we did not have these kinds of separated tests data from Mathematics II. The results are shown in Table 2.

\begin{tabular}{l|c|c|c}
\hline Value & $\boldsymbol{r}$ & $\boldsymbol{t}$ & $\chi^{2}$ \\
\hline calculated & 0.277 & 2.070 & 21.696 \\
\hline from literature & $0.208(p<0.01)$ & $2.609(p<0.01)$ & $21.666(p<0.01)$ \\
\hline
\end{tabular}

Table 2. Statistical comparison marks of theoretical and practical tests

As the data in Table 2 show, the correlation coefficients are similar to those in Table 1, since we got the values from the same students' group. Executing the Student test, we can say that there is no significant difference between the two sets of data at $1 \%$ error level. According to the $\chi^{2}$ test results the data are not significantly different, at $1 \%$ error level.

Note that we had to pay attention to the pedagogical equivalency of the exercises. Despite the exercises to be solved obviously were harder, the subject matter was more than those were in the traditional chalk-and-talk teaching form, the results became better. Examination of dependence of achievement levels on the type and difficulty of exercises needs larger number of students, so it needs further research.

\subsection{The students' assessment of the teaching strategy}

At the end of each semester the students evaluated the new teaching-learning method. Nobody dismissed this way of learning totally; however, 7 percents of the students felt that they had problems with this new type of learning, comparing it to the traditional way one.

Figure 9 shows opinion's distribution about using CAS in the teaching-learning process. In the questionnaire there were two negative answer-options ("complicated the understanding with computer problems" and "the English language environment of the program") and three positive ones ("acquitted me of lengthy calculations", "helped the problem-solving by prepared worksheets" and "helped me to understand the complicated exercises"). The positive properties got higher scores. In the engineering practice the long numerical calculations cause a lot of problem. It is a great success that our students ordered the numerical calculations opportunities of CAS into the first place. 

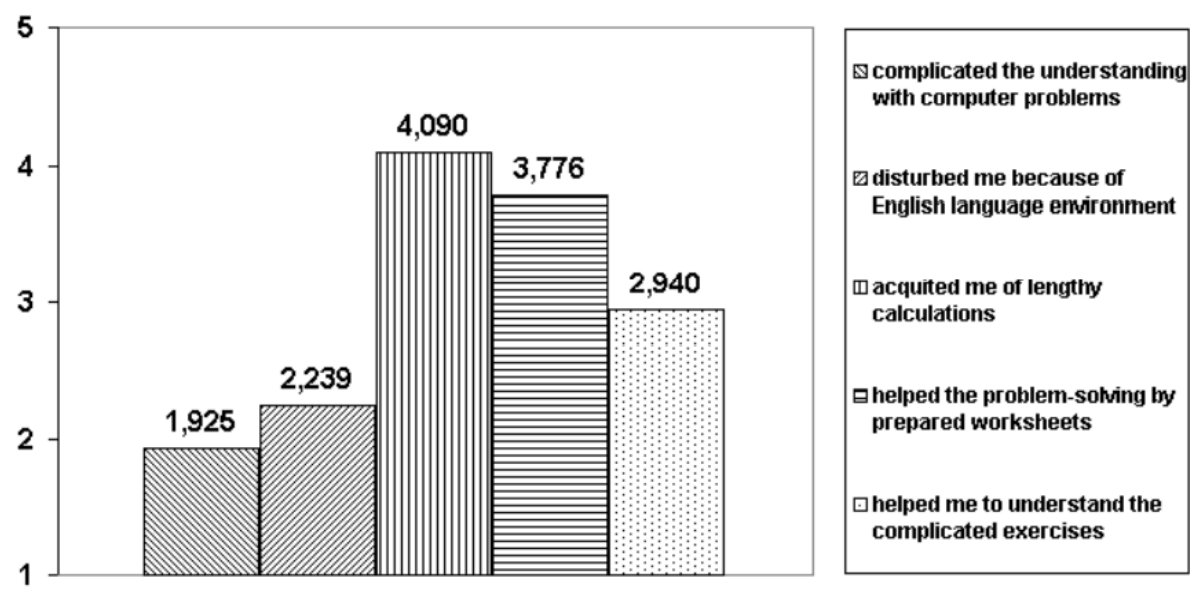

Figure 9. Question: Order the statement about CAS (1 not important, 5 most important)!

We show in Figure 10 that using of the whole web-based syllabus raised the efficiency acquirement of mathematics more than CAS itself. An explanation can be for this fact that the MAPLE computer algebra system meant newer difficulties for those students who are not familiar with computer programming.
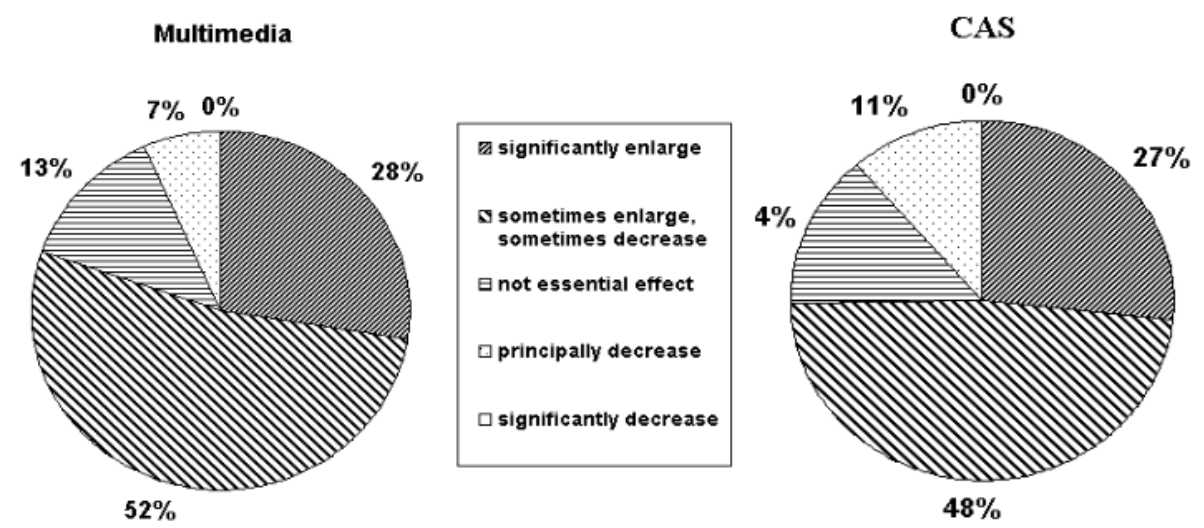

Figure 10. Efficiency of using multimedia and CAS to the Mathematics knowledge acquirement

On Figure 11 are seen the numbering of the listed new elements. The prepared worksheets principally helped the exercise solving. The verbal explanation got the 
second place, which means that the collective problem-solving on the easy exercise without CAS is not negligible. However there is not great difference between the scores, excepting the textbook, which got the lowest score. My explanation is for this that all other elements are free, but the book.

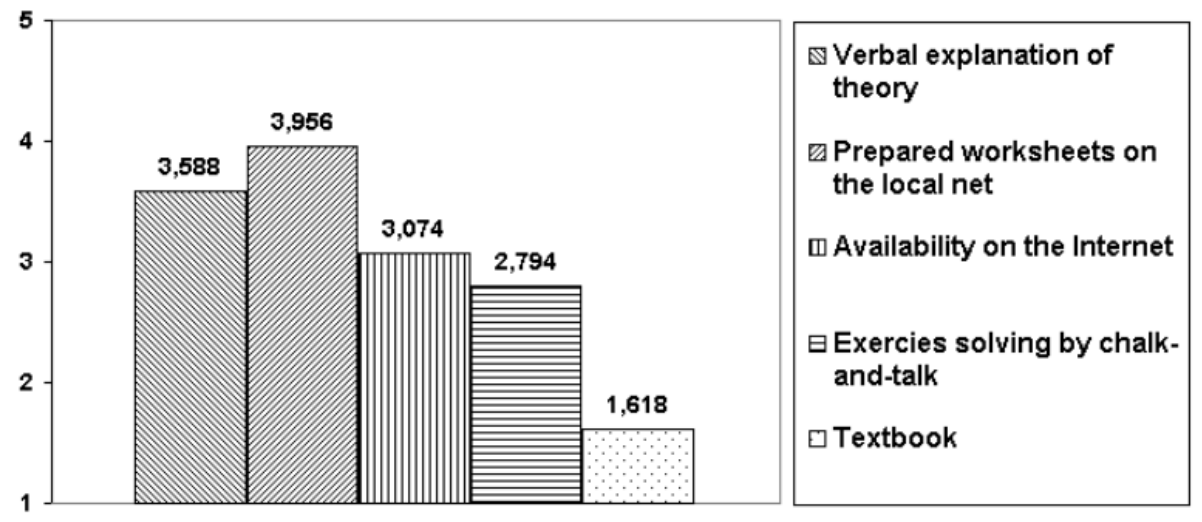

Figure 11. Question: How far were you helped in learning process? (1-low, 5-high)

Figure 12 shows results of the students comparison of mathematics lessons using multimedia, conventional mathematics-, and other engineering-subject-lessons. Although mathematics is the most frightening and unloved subject for engineering students, it is a great result that CAS- and multimedia-based lessons won students' approval.
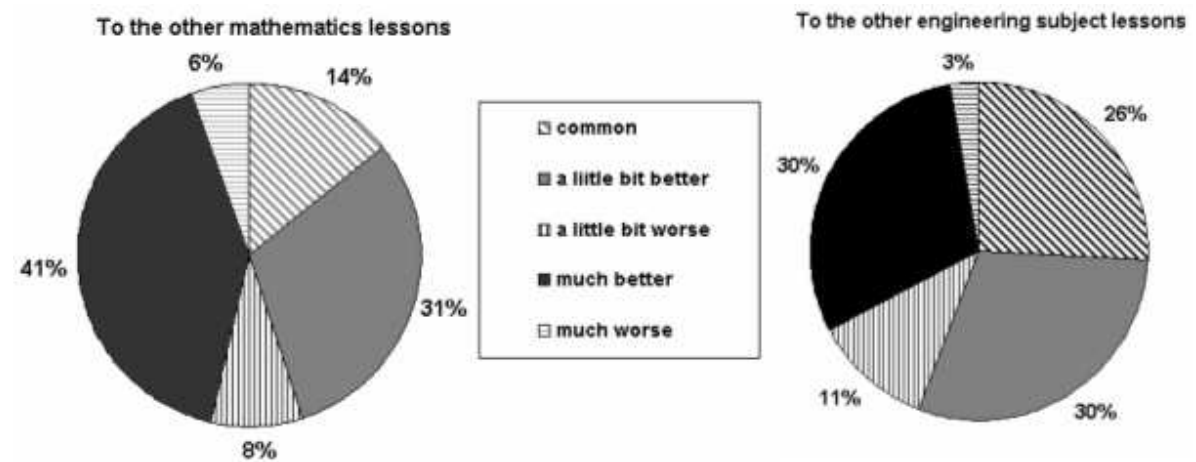

Figure 12. Comparison the mathematics lessons using multimedia to the other mathematics and engineering subject lessons 


\section{Conclusion}

Summarizing the results we can say that the development was successful. The interactive learning environment effectively helped the teaching-learning process. Because of the fast development of informatical tools, however, we have to continuously improve the subject material package.

In more general context, based on our experience we can say that the more and more important role of computers in education needs revision of the whole curriculum of mathematics, both in the middle-school and higher education level.

Introducing computerized mathematical tools into the educational process will be unavoidable in the future. For development of using CAS into the education we have to bring up a new generation of teachers, who are not averse to get to know new methods, while - with critical approach - they assist to develop new educational strategies and try them in the practice.

\section{References}

[1] D. Crowe and Z. Hossein, Computers and undergraduate mathematics 1: setting the scene, Computers \& Education 35 (2000), 95-121.

[2] P. Drijvers, Students encountering obstacles using a CAS, International Journal of Computers for Mathematical Learning 5 (2000), 189-209.

[3] D. Poutney, C. Leinbach and T. Etchells, The issue of appropriate assessment in presence of a CAS, International Journal of Mathematics Education Science and Tecnology 33(1) (2002), 15-36.

[4] K. Aspestsberger, Elemetarization and modularization- two didactical aims being realized by using Computeralgebra Systems, Proceedings of ICTMT4 (1999), 1-9.

[5] C. Leinbach, D. C. Pountey and T. Etchells, Appropriate use of a CAS in the teaching and learning of mathematics, International Journal of Mathematics Education Science and Tecnology 33(1) (2002), 1-14.

[6] J. Karsai, É. Veronika Rácz, A. Schwenk and N. Kalus, Visualization and art in the mathematics classroom, Zentralblatt für Didaktik der Mathematik 35(1) (2003), 24-29.

[7] I. Kidron and N. Zehavi, The role of animation in teaching the limit concept, The International Journal of Computer Algebra in Mathematics Education 9(3) (2002), 205-227.

[8] M. Kawski, How CAS and Visualization lead to a Complete Rethinking of an Introduction to Vector Calculus, Third International Conference on Technology in Mathematics Teaching, Koblenz, 1997. 
[9] A. Kolb and D. A. Kolb, Learning styles, Encyclopedia of Higher Education in the United States, (In Forest, J. \& Kinser, K., eds.), ABC-CLIO Publishers, 2001.

[10] I. Perjési-Hámori and M. Klincsik, An Interactive Method of Teaching Vectorcalculus via Internet, ODL Networking for Quality Learning, Conference Proceedings, Lisbon, 2000, 67-71.

[11] Vectorcalculus for mechanical engineering students, URL: http://matek.pmmf.hu.

[12] M. Klincsik and I. Perjési-Hámori, Vectorcalculus with technological physical and MAPLE applications, University Press, Pécs, 1999.

ILDIKÓ PERJÉSI-HÁMORI

INSTITUTE OF MATHEMATICS

POLACK MIHÁLY ENGINEERING FACULTY

UNIVERSITY OF PÉCS

H-7628 PÉCS BOSZORKÁNY U. 2.

HUNGARY

E-mail: perjesi@witch.pmmf.hu

(Received July, 2003) 